\title{
La recuperación de los envases de hojalata de los RSU en España: calidad
}

\section{de la chatarra}

\author{
A. López-Delgado*, C. Peña*, V. López*, M.A. Lobo-Recio* y F.A. López*
}

Resumen Los envases de hojalata procedentes de RSU se recuperan y reciclan con mayor facilidad que otros materiales gracias a sus propiedades magnéticas. El acero empleado para la fabricación de este tipo de envases es de alta calidad, por lo tanto, tras su utilización se recupera una chatarra de calidad, cuyo reciclado supone una oportunidad importante para reducir el consumo de materias primas. En este trabajo, se analizan los escenarios de la recuperación de envases de hojalata de los RSU en España, se caracteriza la chatarra férrica procedente de plantas de incineración y de compostaje estableciéndose la relación calidad/tecnología de recuperación y se ensayan técnicas de fragmentación/separación magnética/fundido y de desestañado térmico para conseguir la mejora de la calidad de las chatarras previa a su reutilización.

Palabras clave Reciclado hojalata. Calidad chatarra. Incineración. Compostaje.

\section{The recovery of packaging steel from municipal solid waste in Spain: the scrap quality}

\begin{abstract}
Steel packaging from municipal solid waste (MSW) is more advantageously recovered than other packaging materials thanks to its magnetic properties. Steel for packaging is of very high quality and accordingly, post-consumer waste produces a high grade ferrous scrap, furthermore, recycling is an important issue for reducing raw material consumption. This work presents a study on recovery of packaging steel from MSW in Spain. The quality of the recovered packaging steels is related with the technologies employed, and the characterization of ferrous scrap from incineration and compost plants is included. Mechanical procedures such as fragmentation/magnetic separation/granulometric classification, and thermal detinning are essayed to allow an enhancement in the quality of scrap previously its reuse.
\end{abstract}

Keywords Scrap recycling. Ferrous scrap quality. Incineration. Compost plant.

\section{INTRODUCCIÓN}

Los envases de hojalata procedentes de los residuos sólidos urbanos (RSU) se recuperan y reciclan con mayor facilidad que otros materiales gracias a sus propiedades magnéticas. El acero empleado para la fabricación de este tipo de envases es de alta calidad, por lo que, en consecuencia, la chatarra recuperada será de alta calidad ${ }^{[1]}$. La utilización de estas chatarras como materia prima en horno eléctrico de arco supone una reducción importante del consumo de materias primas y de energía con respecto a la fabricación a partir de mineral de hierro.
Básicamente, los envases de hojalata están formados por una lámina de acero bajo en carbono revestida por ambas caras con una película de estaño depositado electroquímicamente y recubierto, a su vez, por una capa de barnices o lacas, las cuales dificultan la corrosión del envase y evitan la dilución parcial del hierro y el estaño en los alimentos contenidos en el mismo.

En España, la fabricación de envases de hojalata ascendió en el año 2001 a 519.000 t, de las cuales, 289.000 t se destinaron al mercado nacional. El reciclado de estos envases supone su recuperación a partir de los RSU, en los que su contenido

(*) Centro Nacional de Investigaciones Metalúrgicas. CSIC Avda. Gregorio del Amo, 8. 28040- Madrid. Email: flopez@cenim.csic.es 
es del orden de $3 \%$ en peso $^{[2}$ y 3$]$. El tratamiento de estos RSU sigue diferentes rutas (Fig. 1), estando actualmente en funcionamiento 62 plantas de compostaje, 9 incineradoras y unas 60 plantas de triaje de recogida selectiva. En el año 2001 se reciclaron $120.586 \mathrm{t}$ de envases de hojalata, lo que equivale a un $42 \%$ de los utilizados, esperándose haber alcanzado un porcentaje de recuperación en torno al $50 \%$ en el año $2002^{\text {[4]. }}$

La calidad de la chatarra férrica procedente de la recuperación de envases de los RSU, en relación con su utilización como materia prima para la fabricación del acero, será mayor cuanto mayor sea su contenido en hierro metálico y cuanto menor sea su grado de oxidación/corrosión y su contenido en elementos indeseados (tramp elements), principalmente cobre y estaño, los cuales afectan negativamente al procesado en caliente del acero ${ }^{[5}$ y 6$]$.

El presente trabajo tiene dos objetivos: el primero de ellos consiste en el estudio de la calidad de la chatarra férrica recuperada de las plantas de incineración y compostaje, el cual, junto con el análisis de los escenarios de la recuperación de los envases de hojalata de los RSU en España, permitirá establecer la relación entre la calidad de la chatarra y la tecnología utilizada para su recuperación; el segundo objetivo es conseguir un aumento sustancial y económicamente viable de la calidad de las chatarras férricas, de manera que éstas puedan ser más eficazmente utilizadas por la industria siderúrgica como materia prima para la fabricación del acero.

Para ello, se han aplicado técnicas de fragmentación, separación magnética y fundido a las chatarras procedentes de plantas de incineración, y téc-

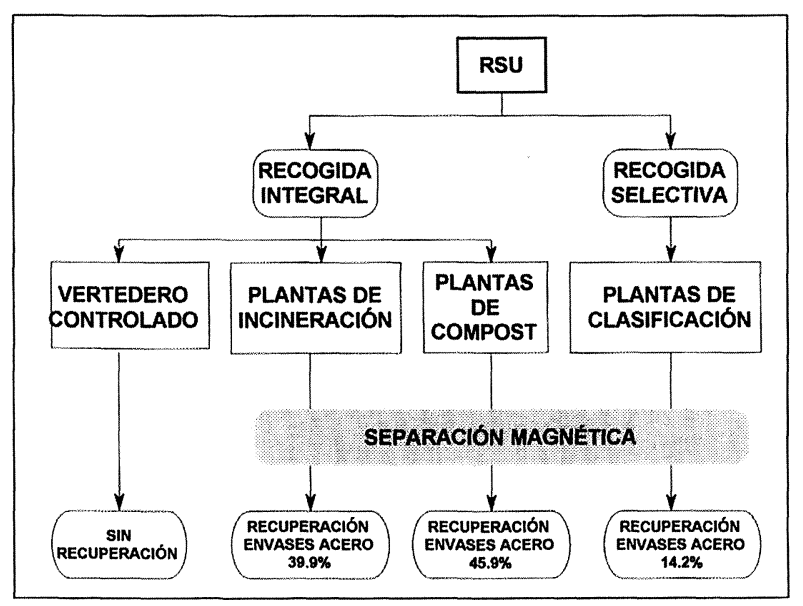

Figura 1. Rutas de tratamiento de los RSU en España.

Figure 1. The management route of MSW in Spain. nicas de limpieza y desestañado térmico a las procedentes de plantas de compostaje.

\section{MATERIALES Y PROCEDIMIENTO EXPERIMEN- TAL}

\subsection{Plantas de incineración}

Las plantas incineradoras de RSU generan, como consecuencia del tratamiento térmico al que éstos son sometidos, una escoria formada por los materiales no combustibles $y / 0$ inertes contenidos en los mismos. Esta escoria, que representa entre el 85 y el $95 \%$ del peso total de los residuos de la combustión, está constituida fundamentalmente por metales y materiales vítreos y cerámicos.

Una vez enfriada, la escoria se somete a una separación magnética, a partir de la cual se obtiene una fracción metálica férrea (chatarra férrica) y una fracción no metálica que contiene los materiales cerámicos, vítreos e inquemados del proceso de combustión (escoria propiamente dicha). Los metales no férreos normalmente no son separados de la escoria, si bien en algunas instalaciones se separa ya el aluminio mediante separadores de Foucault. El porcentaje de fase metálica en la escoria es del $16 \%$, del que la fracción férrea representa el $95 \%$. La producción española de chatarra férrica procedente de la incineración de RSU es de 40.000 t/año.

En el proceso de incineración se utilizan básicamente dos tipos de tecnologías: horno de parrilla móvil (HPM) y horno de lecho fluido (HLF). El primero es el más frecuente, enfriándose con agua la escoria resultante, mientras que en los HLF el enfriamiento se realiza con aire.

Como consecuencia del proceso térmico al que son sometidos los RSU y, sobre todo, de las condiciones de enfriamiento de la escoria (agua o aire), la chatarra férrica obtenida de las plantas de incineración de RSU presenta un elevado grado de alteración química (oxidación/corrosión). Así, tras estos procesos, la chatarra queda recubierta por unos productos de alteración, principalmente óxidos y oxihidróxidos de hierro, que hacen que su calidad, desde un punto de vista metalúrgico, sea baja, presentando en su etapa posterior de reciclado al horno de arco eléctrico inconvenientes importantes, entre ellos, aumento del consumo de reductores, aumento del consumo energético, posibles daños al refractario, presencia de elementos indeseables, generación de humos durante la carga, salpicaduras del baño y aumento de los tiempos de 
colada $^{[7]}$. De ello, se deduce la necesidad de mejorar la calidad de estas chatarras de un modo, tanto tecnológica como económicamente, viable. En este sentido, se describen en este trabajo los resultados del proceso de fragmentación/separación magnética/fusión seguido, con el objetivo de enriquecer en hierro metálico dichas chatarras férricas.

\subsection{Plantas de compostaje}

La chatarra férrica procedente de estas plantas se obtiene fundamentalmente mediante separación magnética y su composición depende, en gran medida, del tipo de tecnología utilizada en las etapas previas de separación de otros RSU (separación manual, tromel, etc.). Esta chatarra férrica muestra menor grado de oxidación/corrosión que la procedente del proceso de incineración, puesto que los RSU no sufren en las plantas de compostaje ningún tratamiento térmico, pero sí presenta un elevado contenido en materia orgánica y en estaño. Por lo tanto, para mejorar su calidad, se hacen necesarios procesos de limpieza y desestañado previos a su posterior reciclado.

\subsection{Estudio de la calidad de la chatarra férrica recuperada}

Se analizaron 12 muestras procedentes de incineradoras y 20 procedentes de plantas de compostaje. Todas las muestras se fragmentaron mediante fragmentadora industrial y cizalla hasta un tamaño aproximado de $20 \mathrm{~mm}$.

Para la caracterización superficial se realizó estudio metalográfico mediante microscopio óptico invertido OLYMPUS, modelo PME 3-312 B.

La composición elemental de la superficie, y en su caso, de los productos de corrosión, se realizó mediante fluorescencia de rayos-X (FRX), en un equipo PHILIPS PW1404 secuencial por dispersión de longitudes de onda.

La composición mineralógica se determinó mediante difracción de rayos-X (DRX) con un difractómetro Siemens modelo D5000. El contenido en estaño de las muestras procedentes de plantas de compostaje se analizó mediante espectroscopia de absorción atómica con un equipo Varian Spectra AA Modelo 220FS.

Para determinar el perfil del recubrimiento de estaño se empleó un espectrómetro de descarga GDL LECO SA 2000.

\subsection{Mejora de la calidad de las chatarras férricas}

Las chatarras férricas procedentes de incineradoras se cortaron mediante fragmentadoras industriales y se cribaron en una criba vibrante por tamiz de $5 \mathrm{~mm}$, obteniéndose así dos fracciones: mayor de $5 \mathrm{~mm}$ y menor de $5 \mathrm{~mm}$. Posteriormente se sometieron a un proceso de separación magnética, utilizando un equipo SMAIS de baja intensidad seco y campo magnético de 1.500 Gauss, obteniéndose fracción magnética y rechazo. La totalidad de la fracción menor de $5 \mathrm{~mm}$ y el rechazo mayor de 5 mm se caracterizaron mediante FRX. La fracción magnética mayor de $5 \mathrm{~mm}$ se fundió en horno de inducción AJAS-GUINEA MAGNETHERMIC de $50 \mathrm{Kg}$ de carga en atmósfera de aire. Se empleó acero de referencia AISI 1010 y silicio como escorificante. El caldo resultante se coló en lingotera, determinando posteriormente la composición del acero. El contenido en azufre (S) y carbono (C) se determinó mediante combustión y posterior detección por absorción en infrarrojo con equipo LECO modelo CS-244.

La limpieza de las muestras de chatarras férricas procedentes de plantas de compostaje se llevó a cabo mediante criba vibrante y disolventes orgánicos para eliminar restos de materia orgánica adherida a las muestras, secándose finalmente con aire a presión.

El análisis térmico se realizó mediante termogravimetría (TG) en una balanza SHIMADZU $50 \mathrm{H}$ a $10^{\circ} \mathrm{C} / \mathrm{min}$, en atmósfera de aire. Este análisis permitió determinar las mejores condiciones para efectuar el desestañado térmico de la chatarra. Para ello se sometieron dos muestras medias a diferentes tratamientos térmicos en un horno con atmósfera de aire: una, a $500{ }^{\circ} \mathrm{C}$ durante $2 \mathrm{~h} \mathrm{y}$, otra, a $700{ }^{\circ} \mathrm{C}$ durante $10 \mathrm{~min}$. Los productos resultantes se caracterizaron química, mineralógica y metalográficamente.

\section{RESULTADOS Y DISCUSIÓN}

\subsection{Chatarra férrica procedente de incinera- ción}

La microestructura observada, de ferrita con cementita terciaria en los límites de grano, es la característica de los aceros de bajo contenido en carbono. El estudio de las muestras por microscopía óptica mostró la presencia de productos de oxidación en caliente y de corrosión del acero en la superficie (Fig. 2). 

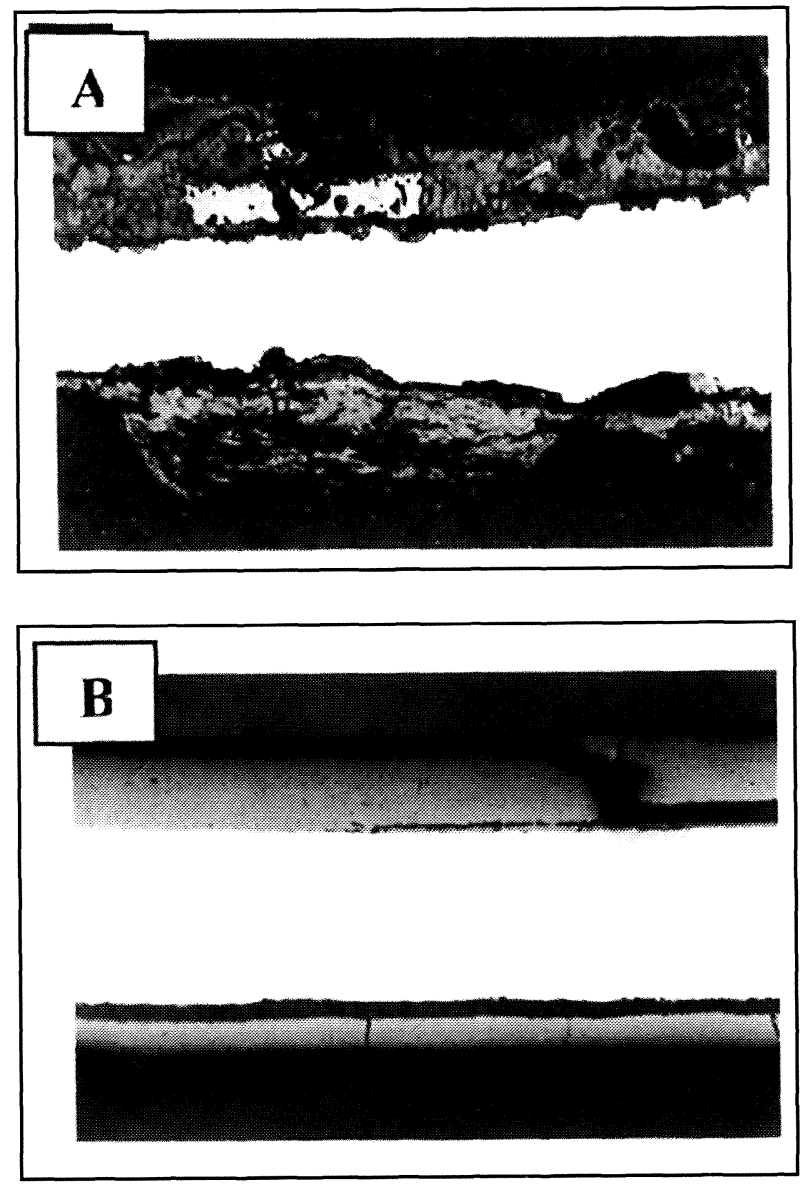

Figura 2. Micrografías a 300 aumentos. (A) corrosión superficial del acero procedente de HPM; (B) capa de oxidación de acero procedente de HLF.

Figure 2. Micrographs (x 300 magnification). (A) superficial corrosion of steel from mobile grate furnace; $(B)$ oxidation layer of steel from fluidised bed furnace.

El proceso de incineración da lugar a la formación de una capa superficial de óxidos de hierro y, al enfriar la escoria, ya sea por agua o por aire, dicha capa sufre rotura o eliminación. Las chatarras procedentes de HLF contienen mayor porcentaje de fase metálica y menor grado de corrosión superficial que las de HPM. Esto se debe al método de enfriamiento de la escoria, ya que es mayor el choque térmico producido por el agua que por el aire, lo que da lugar a la rotura de la capa de oxidación en caliente provocando así la corrosión del acero. El análisis mediante DRX mostró que los productos de corrosión están constituidos, fundamentalmente, por óxidos de hierro tipo hematites, oxihidróxidos de hierro y, en menor proporción, óxidos de silicio, aluminio, calcio y magnesio.

Las técnicas de fragmentación/clasificación granulométrica/separación magnética proporcionaron una fracción magnética total equivalente a la fase magnética de la chatarra inicial con pérdidas de material inapreciables durante el desarrollo de estos procedimientos. Así mismo, se consiguió el descascarillado, mejorando la calidad de la chatarra. En la posterior fusión se produjo la volatilización parcial del estaño (debida a la temperatura alcanzada durante el tratamiento térmico), y una tasa de enriquecimiento en hierro del $87 \%$ para HPM y del $95 \%$ para HLF.

\subsection{Chatarra férrica procedente de plantas de compostaje}

Está constituida fundamentalmente por fracción metálica acompañada de materiales impropios (plástico, papel, etc.) y materia orgánica. El contenido medio en estaño es de 0,43 \%.

La curva TG (Fig. 3) muestra la pérdida de peso producida por la combustión de la materia orgánica de pinturas y barnices que recubren el metal, la cual tiene lugar hasta $543^{\circ} \mathrm{C}$. Entre $575-800^{\circ} \mathrm{C}$ se produce un incremento de peso debido a la oxidación del acero. Es, por esto, que el desestañado se ensayó en dos condiciones: a $500{ }^{\circ} \mathrm{C}$ durante $2 \mathrm{~h}$, para evitar la oxidación de la chatarra resultante y a $700{ }^{\circ} \mathrm{C}$ durante $10 \mathrm{~min}$, para obtener un desestañado más efectivo.

El contenido en estaño determinado mediante absorción atómica resultó de 0,16 \% en el primer caso, e inferior a 0,05\% en el segundo. Mediante un estudio metalográfico, se compararon, a modo de referencia, las muestras después del proceso del calentamiento para el desestañado con materiales no tratados térmicamente (Fig. 4).

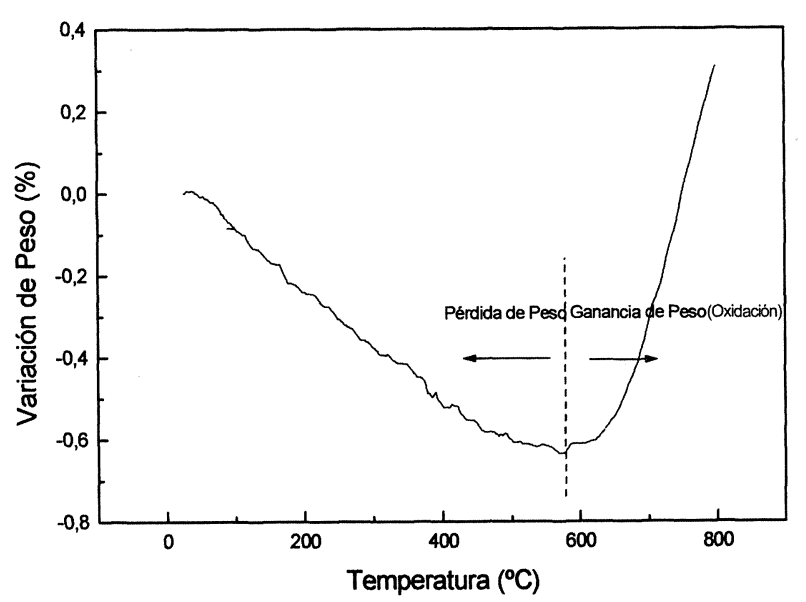

Figura 3. Curva GT de hojalata procedente de planta de compostaje.

Figure 3. TG curve of steel packaging from compost plant. 


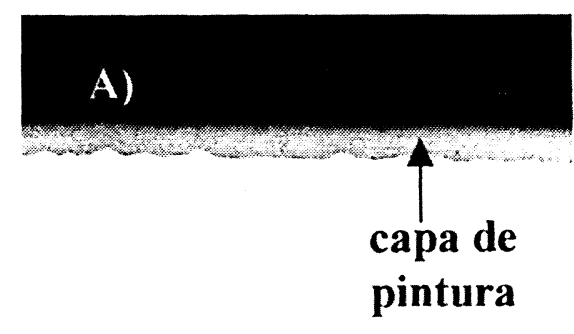

ACERO
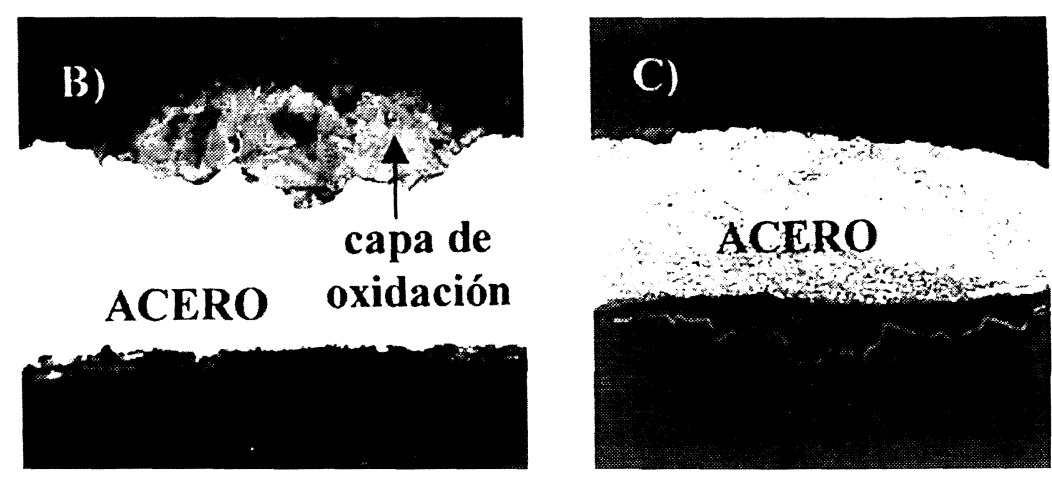

Figura 4. Micrografías (x 500) envase de hojalata. (A) muestra de referencia; (B) tratamiento térmico a $500^{\circ} \mathrm{C} ;(\mathrm{C})$ tratamiento térmico a $700^{\circ} \mathrm{C}$.

Figure 4. Micrographs $(\times 500)$ steel packaging. (A) reference sample; (B) thermal treatment at $500{ }^{\circ} \mathrm{C}$; (C) thermal treatment at $700^{\circ} \mathrm{C}$.

Los difractogramas de las muestras desestañadas presentaron rutilo $\left(\mathrm{TiO}_{2}\right)$, procedente de la descomposición de las pinturas y barnices que recubren a este tipo de envases metálicos. Sin embargo, los oxihidróxidos de hierro, presentes tras el calentamiento a $500{ }^{\circ} \mathrm{C}$, no aparecieron a $700{ }^{\circ} \mathrm{C}$. La caracterización del recubrimiento de estaño mediante GDL mostró una capa externa de 1,98 $\mathrm{g} / \mathrm{m}^{2}$ con una profundidad de $271,4 \mathrm{~nm}$ para la muestra de referencia, mientras que en la muestra tratada a $500{ }^{\circ} \mathrm{C}$ esta profundidad disminuyó a 20 $\mathrm{nm}$, no detectándose recubrimiento de estaño en la muestra calentada a $700{ }^{\circ} \mathrm{C}$.

\section{CONCLUSIONES}

Este estudio ha permitido determinar la composición de diferentes muestras de residuos de envases metálicos, procedentes de procesos de incineración y de plantas de compostaje de RSU.

La calidad de la chatarra férrica proveniente de incineración depende, especialmente, del proceso empleado para el enfriamiento de la escoria. $\mathrm{La}$ chatarra procedente de HLF, donde el enfriamiento se realiza por aire, presenta mayor calidad (mayor contenido en hierro, menor degradación superficial) que la procedente de HPM (enfriamiento por agua).

Procedimientos de fragmentación, clasificación granulométrica y separación magnética conducen a la mejora de la calidad de la chatarra.

La chatarra férrica procedente de plantas de compostaje presenta un contenido en estaño relativamente alto y va acompañada de materia orgánica y otros impropios que disminuyen su calidad, por lo que se hace necesario un tratamiento de limpieza y desestañado previo a su reutilización.

Las condiciones idóneas para el desestañado térmico de estas chatarras implican temperaturas relativamente altas $\left(700^{\circ} \mathrm{C}\right)$ y tiempos cortos de residencia en el horno (10 $\mathrm{min})$. Se consigue así eliminar casi el $100 \%$ del estaño y la obtención de chatarras con un muy bajo grado de oxidación superficial y ausencia prácticamente total de corrosión.

\section{Agradecimientos}

Los autores agradecen a ECOACERO S.A. su colaboración y financiación en este estudio, y a D. Antonio Delgado su asistencia técnica.

\section{REFERENCIAS}

[1] R. Boom y R. Steffen, Steel Res. 72 (2001) 91-96.

[2] F.A. López, C. PeÑa y A. López-Delgado, Recycling and Waste Treatment in Mineral and Metal Processing, vol. 2, Luleå, Sweden, 2002, B. Björkman, C. Samuelsson y J.O. Wikström (Eds.), GTC Print AB, Luleå, Sweden, 2002, pp. 169-178.

[3] A. López-Delgado, C. Peña, V. López y F.A. López, Resource Conservation and Recycling (2003) Pruebas de imprenta.

[4] F.A. López, C. PeÑA y A. López-Delgado, $7^{\text {th }}$ Eur. Electr. Steelmaking Conf., Venice, Italy, 2002.

[5] D. JANKE y L. SAVOV, Resource for Tomorrow-Materials Recycling 294 (1998) 49-66.

[6] L. Savov, S. Tu y D. JankE, ISIJ Int. 40 (2000) 654-663.

[7] N. Sano, H. Katayama, M. Sasabe y S. Matsuoka, Scand. J. Metall. 27 (1998) 24-30. 